\title{
Impacto de la mortalidad prematura en los años de vida saludable perdidos por diabetes en Cuba. Periodo 1990-2015
}

\author{
Impact of premature mortality on disability-adjusted life years \\ for diabetes in Cuba. Period 1990-2015
}

\author{
Emma Domínguez-Alonso ${ }^{*}$ y Oscar Díaz-Díaz ${ }^{2}$ \\ ${ }^{1}$ Departamento de Investigaciones; ${ }^{2}$ Centro de Atención al Paciente Diabético. Instituto de Endocrinología, La Habana, Cuba
}

\section{RESUMEN}

Objetivo: Describir la tendencia del impacto de la mortalidad prematura en las pérdidas de vida saludable como consecuencia de la diabetes mellitus en Cuba en el periodo 1990-2015. Métodos: Estudio de extensión nacional. Los años de vida saludable perdidos se obtuvieron como la suma de los años de vida potencial perdidos por mortalidad prematura y los años de vida perdidos por morbilidad. Los primeros se calcularon a partir del total de defunciones del país en que la diabetes fue consignada como causa básica; y los segundos como el producto de la severidad asignada a la diabetes en estudios similares, y de la incidencia y la duración promedio, esas dos últimas estimadas mediante el programa DISMOD. Adicionalmente, se obtuvieron indicadores que permitieron describir la tendencia de la edad de los fallecimientos. Resultados: La tasa de años de vida saludable perdidos se incrementó del año 1990 al 2015 , de 5.9 a 11.2 por 1,000 en los hombres y de 11.2 a 18.8 por 1,000 en las mujeres, a expensas de las pérdidas por morbilidad (ascensos de la tasa de 2.5 a 8.4 y de 4.3 a 14.8 por 1,000, respectivamente). La tasa de años de vida perdidos por mortalidad prematura descendió en el periodo. La media de la edad de los fallecidos se elevó en ambos sexos, lo que resultó consistente con un ascenso del porcentaje de defunciones ocurridas en edades de 85 años y más del 9.9 al $18.2 \%$ de 1990 a 2015 . Conclusiones: El incremento de los años de vida saludables perdidos debido a la diabetes en Cuba en el periodo 1990-2015 fue a expensas de la morbilidad y no de la mortalidad prematura.

Palabras clave: Años de vida saludable perdidos. Diabetes. Cuba. Años de vida perdidos por mortalidad prematura. Años de vida perdidos por morbilidad.

\begin{abstract}
Objective: Describe the trend of the impact of premature mortality on the disability-adjusted life years for diabetes in Cuba in the period 1990 to 2015. Methods: A nationwide study was conducted. Disability-adjusted life years were obtained from the sum of potential years of life lost plus years lived with disability. The first is calculated based on total deaths in the country in which diabetes was recorded as underlying cause; the second from the product of severity, as assigned to diabetes in similar studies, and incidence and average duration, provided by the DISMOD II program. Additionally, indicators were obtained to describe the trend of the age of deaths. Results: The rate of disability-adjusted life years increased from 5.9/1000 in 1990 to $11.2 / 1000$ in 2015 in men and from 11.2 to $18.8 / 1000$ in women, due to morbidity (increases in the rate of 2.5 to 8.4 and 4.3 to $14.8 / 1000$, respectively). The rate of years of life lost due to premature mortality decreased in the period. The mean age of the deceased rose in both sexes, which was consistent with an increase in the percentage of deaths that occurred in ages 85 and over, from $9.9 \%$ in 1990 to $18.2 \%$ in 2015. Conclusions: The increase disability-adjusted life years for diabetes in Cuba in the period 1990-2015, was at the expense of morbidity and not of premature mortality.
\end{abstract}

Key words: Diabetes mellitus. Cuba. Disability-adjusted life years. Potential years of life lost. Years lived with disability.
Correspondencia:

*Emma Domínguez-Alonso
E-mail: emmadadinfomed.sld.cu
Fecha de recepción: 28-10-2020

Fecha de aceptación: 19-02-2021

DOI: 10.24875/ALAD.20000036
Disponible en internet: 17-03-2021 Rev ALAD. 2021;11:53-62

2248-6518 / ๔ 2021 Asociación Latinoamericana de Diabetes. Publicado por Permanyer. Este es un artículo open access bajo la licencia CC BY-NC-ND (http://creativecommons.org/licenses/by-nc-nd/4.0/). 


\section{INTRODUCCIÓN}

La prevalencia de diabetes mellitus (DM) ha mostrado un incremento catalogado como epidémico a partir de las últimas décadas de la pasada centuria, que se ha atribuido al envejecimiento poblacional y a los cambios de estilos de vida asociados a la urbanización ${ }^{1,2}$.

La Federación Internacional de Diabetes (IDF, por sus siglas en inglés) reportó un $9.3 \%$ de los adultos de 20 a 79 años en el mundo viviendo con DM en el año 2019. La cifra pronosticada para 2045 es del $10.9 \%$, con diferencias entre regiones, que fluctúan del 15 al $143 \%$ en Europa y África, respectivamente 3 .

La diabetes mellitus tipo 2 (DMT2) aporta alrededor del $90 \%$ de los casos de la enfermedad ${ }^{1}$. El sobrepeso y la obesidad son los mayores predictores de DMT2, por lo que el comportamiento epidémico de la enfermedad está asociado al ascenso de la prevalencia de ambas condiciones durante las últimas décadas ${ }^{4,5}$.

En Cuba, la prevalencia de DM en población general se incrementó de 23.6 a 64.3 por 1,000 del año $2000^{6}$ al 20187. Esta tendencia puede ser atribuida al incremento de la prevalencia de sobrepeso y obesidad ${ }^{8}, \mathrm{y}$ a un acelerado envejecimiento poblacional9.

La disponibilidad de estimados confiables de la magnitud de la carga de la DM es imprescindible para orientar y evaluar las políticas encaminadas a su prevención y control. La metodología de carga de enfermedad ha sido adoptada por numerosos países, al efecto de planificar y evaluar las estrategias en salud.

El primer estudio global de carga de enfermedad (Global Burden of Disease Study [GBD] en la literatura inglesa) fue referido al año 1990 (GBD 1990) ${ }^{10}$.
Este sirvió de base para el desarrollo sistemático de estudios globales posteriores ${ }^{11-13}$.

La metodología del GBD 1990 incluyó la introducción de un nuevo indicador, conocido como años de vida saludables (AVISA) perdidos o años de vida ajustados por discapacidad (AVAD) en la literatura hispana, y disability-adjusted life years (DALYs) en inglés. Este es una medida de tiempo que integra los años de vida (potenciales) perdidos por mortalidad prematura (AVPM) y los años de vida perdidos por vivir en condiciones no óptimas de salud (morbilidad o discapacidad) (AVPD) $)^{10,11}$.

Son escasos los estudios cubanos en los que se ha cuantificado las carga de la DM en términos de AVISA. Estos coinciden en reportar incrementos de las pérdidas de vida saludable consecuentes a la DM en diferentes periodos y grupos poblacionales, a expensas de la morbilidad y no de la mortalidad prematura ${ }^{14-16}$.

Adicionalmente, un estudio reciente reportó que la DM ocupó el primer lugar como causa de pérdidas por morbilidad y el segundo como causa de AVISA entre ocho enfermedades crónicas analizadas durante el trienio 2013-2015 ${ }^{17}$.

La tendencia de la mortalidad por DM en Cuba en las últimas décadas ha mostrado fluctuaciones, con un incremento anual del 0.67\% del año 2001 al $2015^{18}$. En el año 2018, la tasa estandarizada de mortalidad por DM en Cuba fue de 10.2 por 100,0007.

Si bien el envejecimiento poblacional incrementa el riesgo de enfermar y morir por DM, paralelamente, en una población envejecida como la de Cuba, hay una mayor proporción de personas en edades en que se incrementa la probabilidad de muerte por la mayoría de las causas. Por otro lado, existe consenso en que las personas con DM pueden tener una 
expectativa de vida similar a la de las no diabéticas cuando se logra un control metabólico adecuado ${ }^{19,20}$.

Lo anterior evidencia la pertinencia de un análisis más profundo e integral de la tendencia de la mortalidad por DM en Cuba que el derivado de la simple interpretación de las tasas.

\section{MATERIAL Y MÉTODOS}

Se llevó a cabo un estudio epidemiológico, descriptivo y de extensión nacional. Periodo de estudio: años 1990, 1995, 2000, 2005, 2010 y 2015.

\section{Estimación de la carga por mortalidad}

Los AVPM se calcularon a partir del total de defunciones ocurridas en el país en que la DM fue consignada como causa básica, en cada uno de los seis años estudiados. Los datos de mortalidad se obtuvieron de los registros de la Dirección Nacional de Estadística del Ministerio de Salud Pública (DNEMINSAP). El registro de mortalidad de la DNE-MINSAP incluye todas las defunciones del país, clasificadas según causa básica de muerte y otras variables generales (edad, sexo, lugar de residencia y lugar y fecha del fallecimiento).

El cálculo de los AVPM se llevó a cabo utilizando la metodología usual adaptada por la Organización Mundial de la Salud ${ }^{10,11}$.

$$
\operatorname{AVPM}=\sum_{x=0}^{\infty} d_{x}\left(L_{x}\right)
$$

donde $x$ es la edad a la muerte, $L_{x}$ es la esperanza de vida a la edad $x$ y dx es el número de fallecidos a la edad $x$.
Se consideró como límite para la vida los estimados de esperanza de vida (EV) para los periodos correspondientes a cada uno de los años incluidos.

Los códigos de la Clasificación Internacional de Enfermedades (CIE) que se consideraron fueron:

- Años 1990, 1995 y 2000. ClE-9: códigos 2 5002509.

- Años 2005, 2010 y 2015. ClE-10: códigos E10-E14.

Se calcularon las tasas de AVPM estandarizadas (por 1,000 habitantes).

Adicionalmente, se obtuvieron otras métricas que permitieron describir la tendencia de la edad de ocurrencia de las defunciones, estas fueron:

- La media y desviación estándar de la edad de los fallecidos por DM.

- Porcentaje de defunciones por DM por grupos quinquenales de edad

\section{Estimación de la carga por morbilidad}

Los AVPD se obtuvieron como el producto de la incidencia (en número de casos), la duración promedio (ambas consistentes internamente, obtenidas como salidas del programa DISMODII) y la severidad correspondiente a la DM.

El DISMOD es un software libre utilizado con el fin de obtener estimaciones de seis indicadores epidemiológicos consistentes entre sí, para lo que es necesario introducir valores de al menos tres de ellos, provenientes de registros. Los indicadores epidemiológi$\cos$ que estima son: incidencia, prevalencia, 
mortalidad, remisión, edad promedio al inicio y duración promedio. Esta aplicación fue desarrollada en el marco del GBD $1990^{10}$, con el propósito de contar con estimaciones más confiables (en relación con los datos provenientes de registros nacionales y regionales) para el cálculo de la carga por morbilidad ${ }^{21}$.

Los datos aportados como entrada al DISMODII fueron: mortalidad por DM (obtenida del registro de mortalidad de la DNE-MINSAP, antes referido), prevalencia de DM (obtenida del registro de dispensarización de la DNE-MINSAP) y la remisión. La remisión se consideró o por tratarse de una enfermedad no curable.

El registro de dispensarización de la DNE-MINSAP incluye todos los casos con diagnóstico de DM en el país. Estos casos son reportados a la DNE-MINSAP por las unidades asistenciales en el momento en que se hace el diagnóstico.

Los valores de severidad fluctúan en un rango entre o y 1; o representa salud perfecta y 1 muerte. La metodología utilizada para la obtención de la severidad se describe en un estudio previo. En el actual se tomó el valor de severidad resultante del referido estudio $(0.17)^{22}$.

Se calcularon las tasas de AVPD estandarizadas (por 1,000 habitantes).

\section{Estimación de la carga integral en términos de años de vida saludables perdidos}

Los AVISA se obtuvieron como la suma de los AVPM y los AVPD. Se calcularon las tasas de AVISA estandarizadas (por 1,000 habitantes) y la proporciones de los AVISA correspondientes a la morbilidad y la mortalidad.
La estandarización de las tasas de AVPM, AVPD y AVISA se llevó a cabo mediante el método directo, tomando la población de Cuba (año 2015) como población estándar.

\section{Aspectos éticos}

La investigación se llevó a cabo a partir de datos provenientes de registros, sin incluir ninguna variable de identificación de los sujetos. Los datos primarios fueron obtenidos de la DNE-MINSAP, lo que se realizó previa autorización de los directivos de la institución.

La investigación fue aprobada por el Comité de Ética de la Investigación del Instituto de Endocrinología.

\section{RESULTADOS}

La figura 1 muestra las tasas de AVISA para los seis años estudiados por sexo. La evolución de 1990 a 2015 es ascendente en ambos sexos; sin embargo, ese comportamiento no es homogéneo para todo el periodo. A partir del año 2005 la tendencia es ascendente de manera consistente. Se evidencia una marcada diferencia entre sexos. El sexo femenino muestra mayores pérdidas de AVISA debido a la DM durante los seis años estudiados.

Los AVISA desagregados en sus dos componentes (AVPM y AVPP) por sexo para los seis años se muestran en la tabla 1. La tasa de AVPM evoluciona de manera descendente del año 1990 al 2015. La disminución más notoria ocurre del año 1995 al 2000 en ambos sexos, a partir de ese año se evidencia un comportamiento estable, con ascensos y descensos muy discretos. Lo contrario ocurre con la tasa de AVPD, con tendencia creciente en ambos sexos, evidente a partir del año 2000 (Tabla 1). 


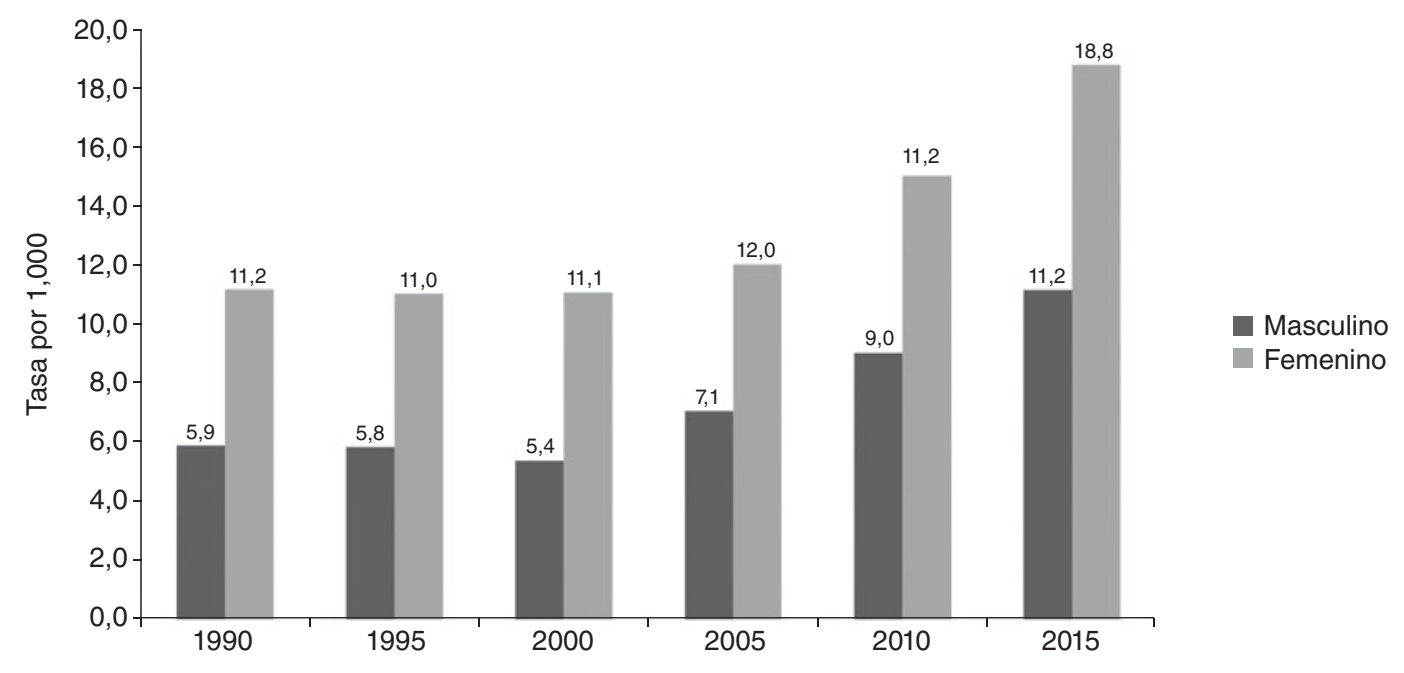

Fıgura 1. Años de vida saludable perdidos* debido a la diabetes mellitus. Cuba: años 1990, 1995, 2000, 2005, 2010 y 2015. *Tasa estandarizada por 1,000.

TABLA 1. Años de vida perdidos por mortalidad prematura (AVPM) y por morbilidad (AVPD) debido a la diabetes. Cuba: años 1990, 1995, 2000, 2005, 2010 y 2015

\begin{tabular}{|c|c|c|c|c|}
\hline \multirow{2}{*}{ Año } & \multicolumn{2}{|c|}{ AVPP por mortalidad* } & \multicolumn{2}{c|}{ AVPP por morbilidad* } \\
\cline { 2 - 5 } & Masculino & Femenino & Masculino & Femenino \\
\hline 1990 & 3.4 & 6.9 & 2.5 & 4.3 \\
\hline 1995 & 3.7 & 6.6 & 2.2 & 4.5 \\
\hline 2000 & 2.0 & 4.1 & 3.4 & 6.9 \\
\hline 2005 & 2.3 & 4.3 & 4.8 & 7.7 \\
\hline 2010 & 2.8 & 4.8 & 6.2 & 10.3 \\
\hline 2015 & 2.7 & 4.0 & 8.4 & 14.8 \\
\hline
\end{tabular}

*Tasa estandarizada por 1,000.

Se reitera la mayor afectación del sexo femenino con respecto al masculino. De modo que las mayores tasas de AVISA en las mujeres (Fig. 1) se corresponden con mayores tasas de los dos componentes (AVPM y AVPD) (Tabla 1).

Las figuras 2 y 3 muestran las proporciones de los AVISA correspondientes a la mortalidad y la

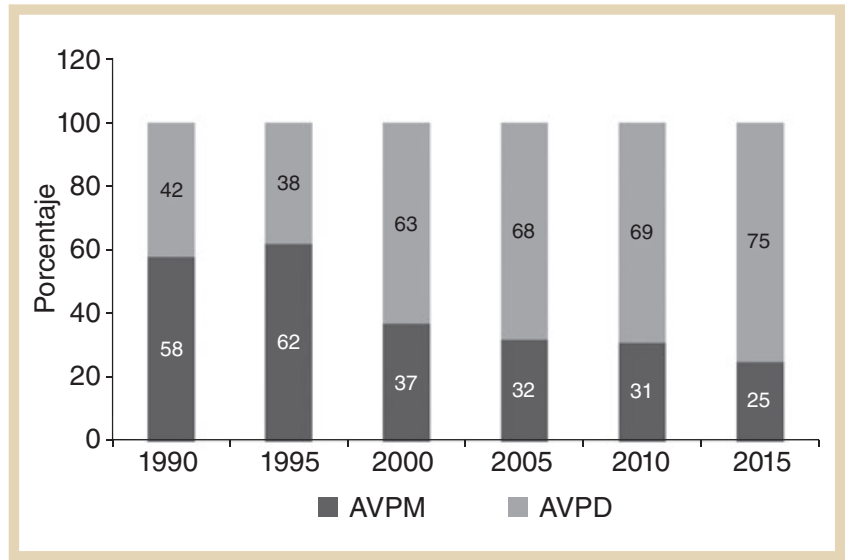

Figura 2. Aporte de la mortalidad prematura (AVPM) y la morbilidad (AVPD) a los años de vida saludable perdidos por diabetes en el sexo masculino. Cuba: 1990, 1995, 2000, 2005, 2010 y 2015.

morbilidad para los seis años en hombres y mujeres, respectivamente.

Se observa una tendencia al incremento del aporte de la morbilidad, con el consecuente descenso de la mortalidad prematura. El aporte de la mortalidad prematura a los AVISA disminuye del 58 al $25 \%$ y del 62 al 21\% del año 1990 al 2015 en hombres y mujeres, 


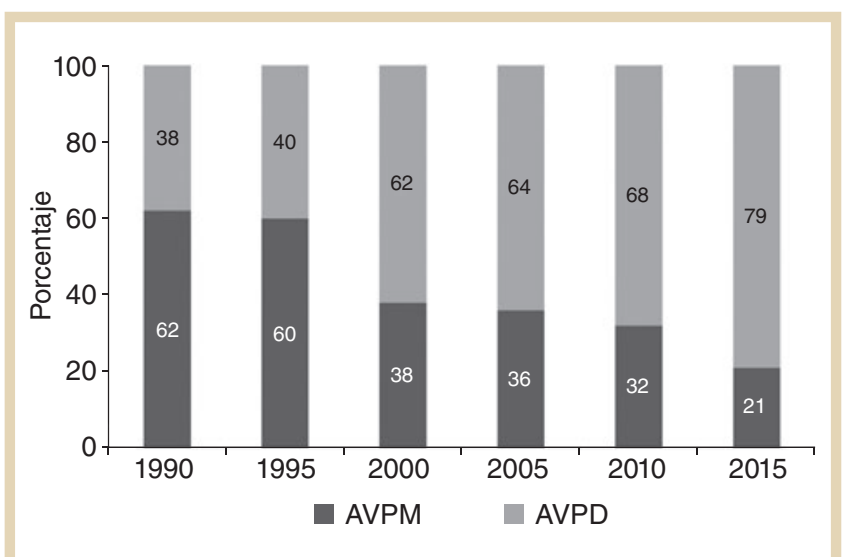

Figura 3. Aporte de la mortalidad prematura (AVPM) y la morbilidad (AVPD) a los años de vida saludable perdidos por diabetes en el sexo femenino. Cuba: 1990, 1995, 2000, 2005, 2010 y 2015.

respectivamente. En consistencia con lo descrito en la tabla 1, el mayor aporte de la morbilidad se hace evidente partir del año 2000, con incremento en los años posteriores en ambos sexos.

La edad promedio de las defunciones por DM aumenta del año 1990 al 2015. Esa tendencia se manifiesta, de manera consistente, a partir del año 2005 en ambos sexos. En los años anteriores la evolución es irregular. Un aspecto de interés es que a partir del propio año se observa una disminución de la variabilidad de la edad de los fallecimientos (menores valores de desviación estándar) en ambos sexos. La media de la edad de las defunciones es mayor en el sexo femenino para todos los años (Tabla 2).

En la figura 4 se representa la distribución porcentual del total de defunciones por DM por grupos quinquenales de edad para los años 1990 y 2015. Se observa un porcentaje mayor de defunciones en los intervalos de edad por debajo de los 65 años en el año 1990 en relación con el año 2015. En el año 1990 el pico de mortalidad ocurre en el grupo de 75 a 79 años de edad, mientras que en el año 2015 se produce en el grupo de 85 años y más. Los porcentajes de defunciones en ese grupo
TABla 2. Media y desviación estándar de la edad de los fallecimientos por diabetes. Cuba: años $1990,1995,2000,2005,2010$ y 2015

\begin{tabular}{|c|c|c|c|c|}
\hline \multirow{2}{*}{ Año } & \multicolumn{2}{|c|}{ Masculino } & \multicolumn{2}{c|}{ Femenino } \\
\cline { 2 - 5 } & Media & DE & Media & DE \\
\hline 1990 & 67.5 & 15.0 & 69.3 & 13.9 \\
\hline 1995 & 65.9 & 16.4 & 70.5 & 14.1 \\
\hline 2000 & 65.7 & 14.9 & 68.8 & 14.2 \\
\hline 2005 & 68.0 & 14.0 & 71.2 & 13.1 \\
\hline 2010 & 70.2 & 13.0 & 72.2 & 12.9 \\
\hline 2015 & 70.5 & 12.9 & 73.6 & 12.7 \\
\hline
\end{tabular}

DE: desviación estándar.

fueron del 9.9 y el $18.2 \%$ en 1990 y 2015 , respectivamente.

\section{DISCUSIÓN}

La tendencia creciente de la tasa de AVISA por DM en Cuba del año 1990 al 2015 es consistente con la situación global. La DM ascendió del lugar 29 entre 107 enfermedades en el año $1990^{10}$ al 9 entre 354 enfermedades en $2017^{12}$ como generadora de pérdidas de vida saludable.

Si bien la evolución de la tasa de AVPM para el periodo (1990-2015) es descendente, el mayor decrecimiento se observa en el año 2000, a partir del cual se evidencian discretos incrementos para algunos años. Un análisis simplista de la evolución de este indicador podría concluir que la mortalidad prematura por DM ha mostrado ligeros incrementos en los últimos años; sin embargo, se precisa un análisis más exhaustivo.

La metodología utilizada para calcular los AVPM en este trabajo, en consistencia con la de los estudios de carga de enfermedad a nivel internacional, es a punto de partida de la EV a la edad de ocurrencia 


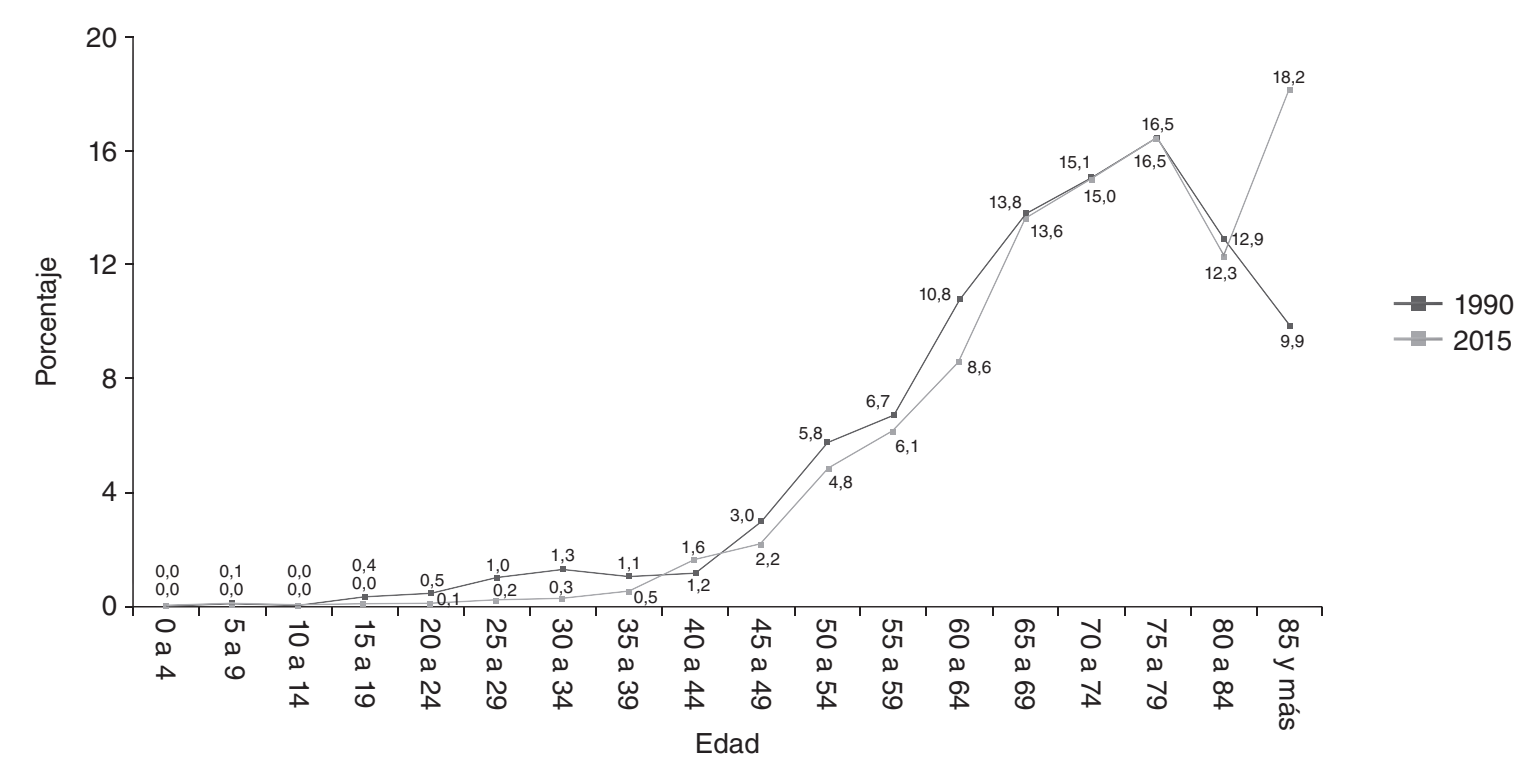

Figura 4. Distribución del total de defunciones por diabetes por grupos quinquenales de edad. Cuba: años 1990 y 2015.

de los fallecimientos ${ }^{10,11}$. De modo que defunciones en edades avanzadas pueden generar pérdidas de vida potencial cuando la expectativa de vida es alta.

Esta situación se corresponde con lo que ocurre en el contexto cubano. Según estimados de la Oficina Nacional de Estadísticas e Información para el periodo 2011-2013, la expectativa de vida de la población cubana a los 65 años era de 17.33 en los hombres y 19.62 en las mujeres ${ }^{23}$.

Por ese motivo se incluyeron otros indicadores, además de los AVPM, para una mejor descripción de la evolución de la edad de los fallecimientos por DM.

El porcentaje de defunciones correspondiente al grupo de 85 años y más es casi el doble en el año 2015 con respecto a 1990, y la edad promedio de los fallecimientos se eleva en el periodo, con los mayores valores en el año 2015. De modo que es posible atribuir la tendencia de la tasa de AVPM en los últimos años a defunciones ocurridas en edades avanzadas.
La situación descrita para Cuba difiere de lo que ocurre a nivel global. Según reporte de la IDF para 2019, el $46.2 \%$ de las defunciones por DM en edades de 20 a 79 años en el mundo ocurrió en personas menores de 60 años $^{3}$.

El ascenso de la tasa de AVPD se corresponde con la situación global. La DM ocupó el cuarto lugar como generadora de AVPD entre todas las causas en el mundo en $2017^{13}$.

El envejecimiento poblacional es un contribuyente fundamental al comportamiento epidémico que ha mostrado la DM en las últimas décadas a nivel global $^{24,25}$.

Cuba ha transitado desde un 11.3 por ciento de personas de 60 años y más en el año 1985 hasta un 20.4 por ciento en el $2018^{9}$.

Otro elemento que considerar en el análisis de la tendencia de la carga de la DM es la relativa a los factores de riesgo para el desarrollo de la 
enfermedad. Existe consenso sobre la influencia de factores como la obesidad y el sobrepeso, la inactividad física y los regímenes alimentarios hipercalóricos de bajo valor nutritivo en el desarrollo de la DMT2 $2^{1,2,4,5}$.

El $40.4 \%$ de la población cubana clasifica como no activa, más de la tercera parte de la población tiene hábitos dietéticos inadecuados. Lo anterior confluye en un $44.8 \%$ de la población con sobrepeso global (incluyendo sobrepeso y obesidad) ${ }^{8}$.

En Cuba, según el presente estudio, a partir del año 2000 el mayor aporte a las pérdidas de vida saludable consecuentes a la DM las hace el componente morbilidad. Esto difiere de lo que ocurre en América Latina y el Caribe (AL-C). Un trabajo que integró y analizó los resultados para la DM del estudio global del año 2015 (GBD 2015) en la región, evidenció que en 27 de los 35 países incluidos en el estudio la contribución de la mortalidad a las pérdidas de vida saludable excede al valor global y es superior al de la morbilidad. Los valores fluctúan desde el 29.7\% (Costa Rica) hasta el $69.3 \%$ (Trinidad y Tobago) ${ }^{26}$.

En el análisis por subregiones llevado a cabo en el propio estudio, el mayor aporte de la mortalidad prematura a los AVISA se identificó en el Caribe (58\%), seguido de Latinoamérica Tropical y Central (55 y 52\%, respectivamente); en todos los casos muy superiores a los reportados por el presente estudio para Cuba en los últimos cuatro años estudiados ${ }^{26}$.

Los aportes de la mortalidad prematura a los AVISA por DM reportados por este estudio, inferiores a los de la región ( $\mathrm{LL}-\mathrm{C}$ ), y muy similares a los de regiones de mayor desarrollo como Europa y países de altos ingresos de Norteamérica y de Asia Pacífico, también reportados en el estudio antes referido ${ }^{26}$, pueden considerarse como un resultado positivo del Programa Nacional de Atención Integral al Paciente
Diabético. Sin embargo, la tendencia creciente de las pérdidas por morbilidad evidencia la necesidad de evaluar y enfatizar las acciones y estrategias encaminadas a la prevención.

El sistema de salud cubano garantiza acceso universal y gratuito a servicios de salud de calidad ${ }^{27,28}$. Este principio, en el caso de la DM, se concreta en el Programa Nacional de Atención Integral al Paciente Diabético, desarrollado en el año 1975, y que ha alcanzado una prioridad creciente a partir de $1992^{29}$.

Otro aspecto que comentar es la mayor afectación en el sexo femenino, lo que es consistente con lo que ocurre en el Caribe, pero diferente de otras regiones ${ }^{30}$.

Las mujeres en el Caribe tienen 2.1 y 2.18 veces mayor riesgo de obesidad y de inactividad física, respectivamente, que los hombres ${ }^{30}$. En Cuba, la prevalencia de inactividad física es mayor en el sexo femenino que en el masculino ( 51 vs. 30\%). Las correspondientes cifras para el sobrepeso son $48.3 \mathrm{vs}$. $41.16 \%{ }^{8}$. Lo anterior, sin duda, constituye un contribuyente esencial para la mayor afectación por DM en las féminas.

Un aspecto de interés es el hecho de que, aunque los actuales resultados confirman la mayor afectación por DM en las mujeres, la media de la edad de las defunciones es mayor en el sexo femenino para los seis años estudiados. Lo anterior es indicativo de que, si bien hay más mujeres que hombres enfermando, y consecuentemente, falleciendo por DM en Cuba, las defunciones ocurren, como promedio, más tardíamente en las primeras que en los segundos.

Una explicación para este hecho puede estar en la mayor solicitud de atención médica por las féminas. El tratamiento y control de la DM se percibe como un fenómeno psicosocial complejo en el que se 
incluyen un conjunto de factores mediadores como el conocimiento, las creencias que el paciente tiene de la enfermedad, la motivación, el estado afectivo y la voluntad para recuperar el control31.

\section{CONCLUSIONES}

El incremento de los AVISA perdidos debido a la DM en Cuba en el periodo 1990-2015 fue a expensas de la morbilidad y no de la mortalidad prematura. La mortalidad por DM en el país se ha desplazado, progresivamente, a edades más avanzadas. Este constituye un aspecto indispensable que tener en cuenta para un análisis exhaustivo de la tendencia de la mortalidad por esta enfermedad en el país. Las mujeres, a pesar de ser más afectadas por la DM en Cuba, mueren más tardíamente que los hombres como consecuencia de esta.

\section{FINANCIAMIENTO}

La presente investigación no ha recibido ninguna beca específica de agencias de los sectores públicos, comercial o sin fines de lucro.

\section{CONFLICTO DE INTERESES}

Los autores declaran no tener conflicto de intereses.

\section{RESPONSABILIDADES ÉTICAS}

Protección de personas y animales. Los autores declaran que para esta investigación no se han realizado experimentos en seres humanos ni en animales.

Confidencialidad de los datos. Los autores declaran que en este artículo no aparecen datos de pacientes.

\section{Derecho a la privacidad y consentimiento informado.} Los autores declaran que en este artículo no aparecen datos de pacientes.

\section{BIBLIOGRAFÍA}

1. Kharroubi AT, Darwish HM. Diabetes mellitus: The epidemic of the century. World J Diabetes. 2015;6(6):850-67.

2. Andersen LB, Grontved A, Moller NC. Worldwide trends in diabetes since 1980. Lancet Oncol. 2016;387(10027):1513-30.

3. International Diabetes Federation. IDF Diabetes Atlas 2019. Bruselas, Bélgica: International Diabetes Federation; 2019 [citado: 28 de enero de 2020 ]. Disponible en: www.idf.org/diabetes mellitus atlas

4. Lean ME, Leslie WS, Barnes AC. Primary care-led weight management for remission of type 2 diabetes (DiRECT): an open-label, cluster-randomised trial. Lancet. 2018;391(10120):541-51.

5. Taylor R, Al-Mrabeh A, Sattar N. Understanding the mechanisms of reversal of type 2 diabetes. Lancet Diabetes Endocrinol. 2019;7:726-36.

6. Dirección Nacional de Estadísticas. Ministerio de Salud Pública. Anuario Estadístico de Salud 2000. Cuba: Dirección Nacional de Estadísticas, Ministerio de Salud Pública; 2001 [citado: 28 de junio de 2020]. Disponible en: http://www.sld.cu/sitios/ dne

7. Dirección Nacional de Estadísticas. Ministerio de Salud Pública. Anuario Estadístico de Salud 2018. Cuba: Dirección Nacional de Estadísticas, Ministerio de Salud Pública; 2001 [citado: 28 de junio de 2020]. Disponible en: http://www.sld.cu/sitios/ dne

8. Bonet-Gorbea M, Varona-Pérez P. III Encuesta Nacional de Factores de Riesgo y Actividades Preventivas de Enfermedades No Transmisibles. Cuba 2010-2011. La Habana: Editorial Ciencias Médicas; 2014.

9. Oficina Nacional de Estadísticas e Información. Centro de Estudios de Población y Desarrollo. El envejecimiento de la población cubana 2018 [Internet]. Cuba: Oficina Nacional de Estadísticas e Información, Centro de Estudios de Población y Desarrollo; 2019 [citado: 12 de julio de 2020]. Disponible en: http://www.one.cu/temaspoblacion.htm

10. Murray CJL, López A. The Global Burden of Disease: a comprehensive assessment of mortality and disability from diseases, injuries and risk factors in 1990 and projected to 2020. Volume 1. Ginebra: World Health Organization; 1996.

11. Murray CJ, Ezzati M, Flaxman AD. GBD 2010: design, definitions, and metrics. Lancet. 2012;380:2063-6.

12. GBD 2017. DALYS and HALE Collaborators. Global, regional, and national disability-adjusted life-years (DALYs) for 359 diseases and injuries and healthy life expectancy (HALE) for 195 countries and territories, 1990-2017: a systematic analysis for the Global Burden of Disease Study 2017. Lancet. 2018;392:1859-922.

13. GBD 2017. Disease and Injury Incidence and Prevalence Collaborators. Global, regional, and national incidence, prevalence, and years lived with disability for 354 diseases and injuries for 195 countries and territories, 1990-2017: a systematic analysis for the Global Burden of Disease Study 2017. Lancet. 2018;392:1789-858.

14. Domínguez Alonso E, Seuc Jo AH, Díaz Díaz O, Aldana Padilla D. La carga de la diabetes en Cuba, período 1990-2005. Rev Cubana Endocrinol. 2008;19(2).

15. Domínguez E, Seuc AH. Años de vida saludable perdidos debido a la diabetes en mujeres cubanas en edad fértil. Periodo 1990-2010. Rev ALAD. 2015;5(3):133-42.

16. Dominguez E. Disability-adjusted life years for diabetes in cuban children and adolescents, 1990-2010. MEDICC Rev. 2016;18(4):14-9. 
17. Corral Martín A, Pría Barros M C, Seuc Jo A H. Desigualdades en la carga global de enfermedad según condiciones de vida. Horizonte sanitario. 2019;18(3):337-46.

18. Landrove-Rodríguez $\mathrm{O}$, Morejón-Giraldoni A, Venero-Fernández S, Suárez-Medina R, Almaguer-López M, Pallarols-Mariño E, et al. Enfermedades no transmisibles: factores de riesgo y acciones para su prevención y control en Cuba. Rev Panam Salud Publica [Internet]. 2018;42:e23. Disponible en: https://doi.org/10.26633/RPSP.2018.23

19. Tachkov K, Mitov K, Koleva Y, Mitkova Z, Kamusheva M, Dimitrova $M$, et al. Life expectancy and survival analysis of patients with diabetes compared to the non diabetic population in Bulgaria. PLoS One. 2020;15(5):e0232815.

20. X He, J Li, B Wang, Q Yao, L Li, R Song, et al. Diabetes self-management education reduces risk of all-cause mortality in type 2 diabetes patients: a systematic review and meta-analysis. Endocrine. 2017;55: 712-31.

21. Barendregt JJ, Van Oortmarssen GJ, Vos T, Murray CJL. A generic model for the assessment of disease epidemiology: The computational basis of DisMod II. Popul Health Metr. 2003;1(1):4.

22. Domínguez E, Seuc AH, Aldana D, Licea M, Díaz O. Impacto de la diabetes sobre la duración y calidad de vida de la población cubana: años 1990, 1995, 2000 y 2003. Rev Cubana Endocrinol. 2006;17(2).

23. Oficina Nacional de Estadísticas e Información. Centro de Estudios de Población y Desarrollo. La esperanza de vida 2011-2013. Cálculos para Cuba y provincias por sexo y edades Mayo 2014 [Internet]. Cuba: Oficina Nacional de Estadísticas e Información, Centro de Estudios de Población y Desarrollo; 05/02/2020. Disponible en: http://www.onei. gob.cu/node/14701

24. Kalyani R, Golden SH, Cefalu WT. Diabetes and aging: Unique considerations and goals of care. Diabetes Care. 2017;40:440-43.
25. Charvat H, Goto A, Goto M, Inoue M, Heianza Y, Arase Y, et al. Impact of population aging on trends in diabetes prevalence: A meta-regres sion analysis of 160,000 Japanese adults. J Diabetes Invest. 2015;6: 533-42.

26. Domínguez Alonso E. La carga de la diabetes en América Latina y e Caribe: análisis a partir de resultados del Estudio Global de Carga de Enfermedad del año 2015. Rev ALAD. 2018;8:81-94.

27. Organización Panamericana de la Salud. Organización Mundial de la Salud. Salud en Las Américas. Cuba. Edición 2012 [Internet]. Organización Panamericana de la Salud, Organización Mundial de la Salud; 2012 [citado: 10 de septiembre de 2018]. Disponible en: http://www. paho.org/salud-en-las-americas-2012/index.php?option=com_content $\&$ view $=$ article $\& i d=32 \% 3$ Acuba $\&$ catid=21\%3Acountry-chap ters\&ltemid=142\&lang=es

28. Domínguez Alonso E, Zacca E. Sistema de salud de Cuba. En: Clérico L, Ronconi L, Aldao M. Tratado de Derecho a la Salud. Parte X, Tomo 3: Derecho a la salud en perspectiva latinoamericana. Buenos Aires: Abeledo Perrot; 2013. pp. 2751-2767.

29. Díaz O, Mateo de Acosta O, Suárez R, García R, Márquez A, et al. Programa Nacional de Diabetes [Internet]. La Habana, Cuba; Infomed, Centro Nacional de Información de Ciencias Médicas; 2000 [citado: 13 de abril de 2018]. Disponible en: http://www.sld.cu/galerias/pdf/ sitios/diabetes/programa_nacional_de_diabetes.pdf

30. Guariguata L, Brown C, Sobers N, Hambleton I, Samuels TA, Unwin $\mathrm{N}$. An updated systematic review and meta-analysis on the social determinants of diabetes and related risk factors in the Caribbean. Rev Panam Salud Publica. 2018;42:e171.

31. Félix P EM, Sombra F GB, Cota G AM, Sarmiento J LG, Félix R EO. Diabetes y género desde una perspectiva sociocultural. Revista Conjeturas Sociológicas. 2019;7(18):130-42. 